\title{
Increased Prevalence of NAFLD in IBD Patients
}

\author{
Jill K. J. Gaidos ${ }^{1} \cdot$ Michael Fuchs ${ }^{1}$
}

Published online: 29 March 2017

(C) Springer Science+Business Media New York (Outside the USA) 2017

Nonalcoholic fatty liver disease (NAFLD), a condition affecting approximately a quarter of the general population, is associated with an enormous clinical and economic burden [1]. The estimated prevalence of NAFLD is as high as $40 \%$ in patients with inflammatory bowel diseases (IBD), including ulcerative colitis (UC) and Crohn's disease (CD) [2]. Although multiple etiologies have been proposed to explain this increased NAFLD prevalence, including the presence of the metabolic syndrome (MetS), intestinal inflammation and dysbiosis, and the medications used to treat IBD [3], the underlying cause(s) have yet to be determined.

In this issue of Digestive Diseases and Sciences, Carr et al. [4] performed a retrospective analysis of 84 patients with NAFLD and IBD (60 CD and $24 \mathrm{UC}$ ), reporting that $23 \%$ had MetS. Compared to the cohort without MetS, the cohort with MetS was diagnosed with both MetS and IBD at an older age, had higher transaminase levels, and had higher NAFLD fibrosis scores, although none had advanced fibrosis. The authors therefore suggest that the presence of MetS in IBD patients should prompt further evaluation for NAFLD by a hepatologist for further evaluation and potential long-term care. There was no significant difference in IBD medication use, IBD severity or disease location, or NAFLD severity between the two study groups.

Michael Fuchs

Michael.Fuchs2@va.gov

Jill K. J. Gaidos

Jill.Gaidos@va.gov

1 McGuire VA Medical Center, GI/Hepatology Service (111N), Virginia Commonwealth University, 1201 Broad Rock Boulevard, Richmond, VA 23249, USA
This study, which contributes new findings to this growing area of research, is limited by its retrospective design and by the use of International Statistical Classification of Diseases and Related Health Problems (ICD)-9 codes for diagnosis, which may have underestimated the prevalence of NAFLD in the IBD population. Furthermore, the absence of a liver biopsy excluded the possibility of diagnosing more advanced liver disease, including nonalcoholic steatohepatitis and fibrosis, which are challenging to diagnose with current imaging modalities. These and other limitations of this study clearly demonstrate the need for rigorously controlled prospective studies to address some of the most pressing questions, which include the potential interrelationship between the gut microbiome, genetic factors, IBD and NAFLD, respectively.

\section{References}

1. Younossi ZM, Blissett D, Blissett R, et al. The economic and clinical burden of nonalcoholic fatty liver disease in the United States and Europe. Hepatology. 2016;64:1577-1586.

2. Bargiggia S, Maconi G, Elli M, et al. Sonographic prevalence of liver steatosis and biliary tract stones in patients with inflammatory bowel disease: study of 511 subjects at a single center. J Clin Gastroenterol. 2003;36:417-420.

3. Chao C-Y, Battat R, Al Khoury A, et al. Co-existence of nonalcoholic fatty liver disease and inflammatory bowel disease: a review article. World J Gastroenterol. 2016;22:727-7734.

4. Carr RM, Patel A, Bownik H, et al. Intestinal inflammation does not predict nonalcoholic fatty liver disease severity in inflammatory bowel disease patients. Dig Dis Sci. (Epub ahead of print). doi:10.1007/s10620-017-4495-0. 